VIEWPOINT

\title{
Managing patients undergoing non-cardiac surgery: need to shift emphasis from risk stratification to risk modification
}

\author{
G Karthikeyan, B Bhargava
}

Many patients undergo non-invasive testing for the detection of coronary artery disease before non-cardiac surgery. This is despite the low predictive value of positive tests in this population and the lack of any evidence of benefit of coronary revascularisation before non-cardiac surgical procedures. Further, this strategy often triggers a clinical cascade exposing the patient to progressively riskier testing and intervention and results in increased costs and unnecessary delays. On the other hand, administration of $\beta$ blockers, and more recently statins, has been shown to reduce the occurrence of perioperative ischaemic events. Therefore, there is a need for a shift in emphasis from risk stratification by non-invasive testing to risk modification by the application of interventions, which prevent perioperative ischaemia-principally, perioperative $\beta$ adrenergic blockade and perhaps treatment with statins. Clinical risk stratification tools reliably identify patients at high risk of perioperative ischaemic events and can guide in the appropriate use of perioperative medical treatment.

See end of article for authors' affiliations

Correspondence to: Dr Ganesan Karthikeyan, Department of Cardiology, Cardiothoracic Sciences Centre, All India Institute of Medical Sciences, New Delhi - 110029 , India karthik_2010@hotmail. com
Heart 2006;92:17-20. doi: 10.1136/hrt.2004.057125

patient was discharged four days later. This case illustrates how a perfectly well meaning sequence of interventions can cascade into catastrophe when inappropriately applied to patients undergoing non-cardiac surgery. Could these events have been avoided?

\section{BACKGROUND}

Most patients undergoing non-cardiac surgery do not suffer adverse cardiac events in the perioperative period. In the oft cited paper by Goldman and colleagues, mortality due to cardiac causes was only $1.9 \%$ in 1001 patients. ${ }^{1}$ Similarly, Lee et al found that major cardiovascular complications occurred in only $2 \%$ of nearly 2900 patients undergoing elective major noncardiac surgical procedures. ${ }^{2}$ More recently, in a high risk population undergoing high risk vascular surgery, Boersma and colleagues found that $83 \%$ of patients had low perioperative event rates irrespective of the results of dobutamine stress echocardiography. ${ }^{3}$ The problem of risk stratification is therefore one of accurately identifying a small number of patients at high risk of cardiac events in a population comprising predominantly of individuals at low risk. This situation renders most strategies that use noninvasive tests for risk stratification inefficient. Despite this, preoperative non-invasive testing is widely advocated. ${ }^{4}$ On the other hand little attention has been focused on the mechanisms responsible for perioperative cardiac ischaemia. Approaches directed at deciphering these mechanisms, and measures to prevent ischaemia, are more likely to be fruitful. Therefore the question which is likely to yield useful answers, is, what causes perioperative cardiac ischaemic events and how these can be prevented, rather than who among the population is at risk. ${ }^{5}$

\section{RISK STRATIFICATION AND THE CASCADE EFFECT: BRER RABBIT KICKS THE TAR-BABY}

Bayesian logic dictates that when the prevalence of disease is low, the strategy used to identify it should be simultaneously highly sensitive and specific. In the context of preoperative evaluation, the typical test is fairly sensitive but not very specific (that is, it has a high false positive rate). Such a test spuriously identifies a large number of patients as being at high risk for

Abbreviations: $C A B G$, coronary artery bypass grafting; $C A D$, coronary artery disease; CASS, coronary artery surgery study; DSE, dobutamine stress echocardiogram; MI, myocardial infarction; NWMA, new wall motion abnormalities not given because of the recent surge Clopidogrel was restarted the next day and the after 24 hours. A day later, the pa Primary angioplasty was complicated by no reflow and poor myocardial perfusion. 
cardiac events. This often triggers the cascade effect, resulting in patients unnecessarily undergoing progressively riskier and costlier testing and intervention. ${ }^{6}$ Not unlike the Brer Rabbit character in the Uncle Remus story, ${ }^{7}$ who progressively gets stuck to the Tar-Baby with every blow starting with the tentative first kick, following an apparently innocuous test, both patient and physician may become helpless victims of a frustrating, runaway situation which might cause more harm than good. Indeed, the hazards of applying additional data to clinical situations have been clearly described. ${ }^{8}$

\section{Clinical variables as reliable predictors of risk}

How then do we stratify risk? A risk stratification tool should be accurate, add significantly to pretest knowledge, and should have a favourable risk-benefit tradeoff. In a recent analysis, risk indices, which used clinical variables, were the only ones which fitted the bill. ${ }^{9}$ Among the many risk indices in clinical use, ${ }^{12}{ }^{10}$ the most simple and yet robust index is the one proposed by Lee and colleagues. ${ }^{2}$ This revised cardiac risk index uses the following six variables to identify patients at high risk: history of ischaemic heart disease, history of heart failure, history of stroke or transient ischaemic attack, preoperative insulin treatment, preoperative serum creatinine values more than $152.5 \mu \mathrm{mol} / \mathrm{l}$, and high risk surgical procedure. The presence of two or more of these risk variables (class III and IV) conferred an event rate as high as $11 \%$ in a group of 1422 patients, whereas the event rate was under $1 \%$ in the presence of one or none of these variables (class I and II). ${ }^{2}$ The performance of this index (area under the receiver operating characteristic curve, 0.806) was significantly better than other indices tested.

\section{Non-invasive stress testing}

The rationale for performing non-invasive stress testing is presumably to simulate the adrenergic stress of surgery and the perioperative period. Although negative predictive values are high, a positive result by any modality of stress testing has a uniformly poor predictive value for major cardiovascular events or death in the perioperative period. ${ }^{9}$ This results in a substantial number of patients being subjected to further evaluation, increased costs, and unnecessary delays in surgery. Why does inducible ischaemia on stress testing not predict perioperative events satisfactorily?

\section{LONG TERM RISK VERSUS PERIOPERATIVE RISK: THE SEARCH FOR MECHANISMS}

In patients with coronary disease, non-invasive testing accurately determines prognosis at intermediate and long term follow up. ${ }^{11}$ The problems of using the results of stress testing to predict perioperative event rates stem from the (inappropriate) extrapolation of prognosis in the intermediate and long term to that over a 96 hour perioperative period. Events accumulated over years cannot be equated to those incurred during a brief, albeit stressful, perioperative period, partly because of the different mechanisms involved. Whereas acute ischaemic syndromes in the setting of chronic coronary artery disease (CAD) occur because of destabilisation and rupture of atherosclerotic plaques, ${ }^{12}$ the pathophysiology of perioperative ischaemic events is not clearly known. Although observations in necropsy specimens of fatal perioperative MI have suggested that about half of these events occur as a result of plaque rupture, ${ }^{13}{ }^{14}$ the predominant belief is that a prolonged state of myocardial oxygen supply-demand imbalance is the culprit. Postoperative pain and intravascular volume shifts also contribute to this imbalance. ${ }^{15}$ In a case-control study, the most common angiographic features which were associated with perioperative MI or death were an occluded coronary vessel subtending viable myocardium followed in frequency by non-obstructive coronary lesions. ${ }^{16}$ Given these considerations, non-invasive testing has the potential to hijack the focus from the noncardiac surgical procedure, which is the primary reason for patient evaluation, to the patient's hitherto stable CAD. This triggers the cascade of events leading on to invasive evaluation and revascularisation before non-cardiac surgery, which is of doubtful benefit.

\section{REVASCULARISATION BEFORE NON-CARDIAC SURGERY: THE HORSE BEHIND THE CART}

It is surprising the number of papers that have evaluated risk stratification with non-invasive stress testing despite the absence of any randomised clinical trial evidence supporting a strategy of revascularisation before non-cardiac surgery. This situation has led some authorities to comment that we have put the cart before the horse. ${ }^{17}$ In a decision analysis model, Mason and colleagues ${ }^{18}$ showed that in patients with a positive non-invasive test for inducible ischaemia (estimated prevalence of CAD of $60 \%$ ) slated to undergo vascular surgery, a strategy of routine catheterisation before surgery resulted in higher mortality, morbidity, and costs. On the other hand, it is clear that patients who have undergone coronary artery bypass grafting (CABG) in the past have lower rates of perioperative events. In a retrospective analysis of the coronary artery surgery study (CASS) database, Eagle and colleagues ${ }^{19}$ showed that in patients undergoing high risk non-cardiac surgery (abdominal, vascular, thoracic, and head and neck surgery), prior CABG resulted in perioperative mortality and MI rates which were 50\% and 70\% lower, respectively. An important limitation of this study was that the mortality and morbidity associated with CABG was not factored into the analysis of perioperative outcomes. This is particularly important because the risk of CABG itself, when added to the subsequent risk of non-cardiac surgery, actually exceeds the risk of non-cardiac surgery in patients who have not undergone prior revascularisation. ${ }^{20}$ Nevertheless there are two important inferences that can be drawn from this study. Firstly, stable patients who have undergone CABG within the last five years should undergo non-cardiac surgery without any further testing. ${ }^{19}$ Secondly, patients undergoing low risk non-cardiac surgery (urologic, orthopaedic, breast, and skin operations) are unlikely to benefit from revascularisation, because as a group they had a mortality of $<1 \%$ regardless of prior coronary treatment. ${ }^{19}$

Percutaneous revascularisation before non-cardiac surgery has again not been evaluated in randomised controlled trials. However, there is evidence for higher adverse event rates in patients undergoing angioplasty before non-cardiac surgery. In a retrospective analysis, Posner and colleagues found that patients who underwent prior angioplasty had perioperative MI and death rates that were not different from those who had not. ${ }^{21}$ But they were more likely to suffer adverse cardiac outcomes if they underwent non-cardiac surgery less than 90 days after angioplasty. ${ }^{21}$ Another recent report highlights the potential for bleeding complications and stent thrombosis if non-cardiac surgery is performed in the weeks following angioplasty. Kaluza and colleagues reported that among 40 patients who had non-cardiac surgery within six weeks of successful stent placement, eight died, seven had non-fatal MI, and 11 had major bleeding episodes. ${ }^{22}$ In the era of drug eluting stents, the spectre of late stent thrombosis after discontinuation of antiplatelet treatment to facilitate noncardiac surgery has been highlighted in the recent report by McFadden and colleagues. ${ }^{23}$ Therefore, current knowledge suggests that there is no role for prophylactic coronary angioplasty, especially with stenting in patients undergoing non-cardiac surgery, and may even be harmful if surgery is performed early after angioplasty. 
A recent randomised controlled trial involving low to moderate risk men showed that revascularisation (CABG and angioplasty) did not alter perioperative or long term outcomes. ${ }^{24}$ Nevertheless, since only a third of these patients had triple vessel disease and most had normal left ventricular systolic function, these results cannot be applied to higher risk patient groups undergoing vascular surgery.

\section{$\beta$ ADRENERGIC BLOCKADE: PREVENTING PERIOPERATIVE ISCHAEMIA}

Given the inefficiency of risk stratification and the futility of prophylactic revascularisation, the logical approach would be to intensify efforts to prevent ischaemia during the perioperative period. Based on the premise that myocardial oxygen supply-demand imbalance is an important cause of perioperative ischaemia, $\beta$ adrenergic blockers have been tested in patients undergoing non-cardiac surgery. Two randomised controlled studies have demonstrated the effectiveness of this strategy. In 200 patients with known CAD or risk factors for atherosclerosis who underwent non-cardiac surgery, perioperative treatment with atenolol resulted in significant reductions in cardiac death in the 6-8 months following the surgical procedure. ${ }^{25}$ This benefit was sustained at two years of follow up. In the other study, 112 patients with one or more cardiac risk factors and a positive dobutamine stress echocardiogram (DSE) result, scheduled to undergo vascular surgery, were randomly assigned either to perioperative bisoprolol or placebo. ${ }^{26}$ In this high risk group, bisoprolol treatment produced a significant reduction in death from cardiovascular causes (3.4\% $v 17 \%)$ and $\mathrm{MI}$ $(0 \% \vee 17 \%)$ at 30 days. In a subsequent analysis, these investigators showed that in patients who were found to be at low or intermediate risk by the revised cardiac risk index (up to two risk factors) and a positive result on DSE, $\beta$ blocker treatment resulted in perioperative event rates similar to those in whom DSE was negative for inducible ischaemia. ${ }^{3}$ Even among patients who were considered high risk by the revised risk index and a positive DSE result, those with new wall motion abnormalities in up to four segments were protected by $\beta$ blocker treatment. Only the small subset of patients ( $2 \%$ of the study population) with new wall motion abnormalities in five or more segments on DSE had high perioperative event rates despite $\beta$ blocker treatment.

The evidence for the perioperative use of other agents ( $\alpha$ agonists like clonidine and mivazerol, and calcium channel blockers) is not robust enough. Considering that a substantial number of perioperative events are presumably caused by plaque rupture, ${ }^{13} 14$ the role of rapid plaque

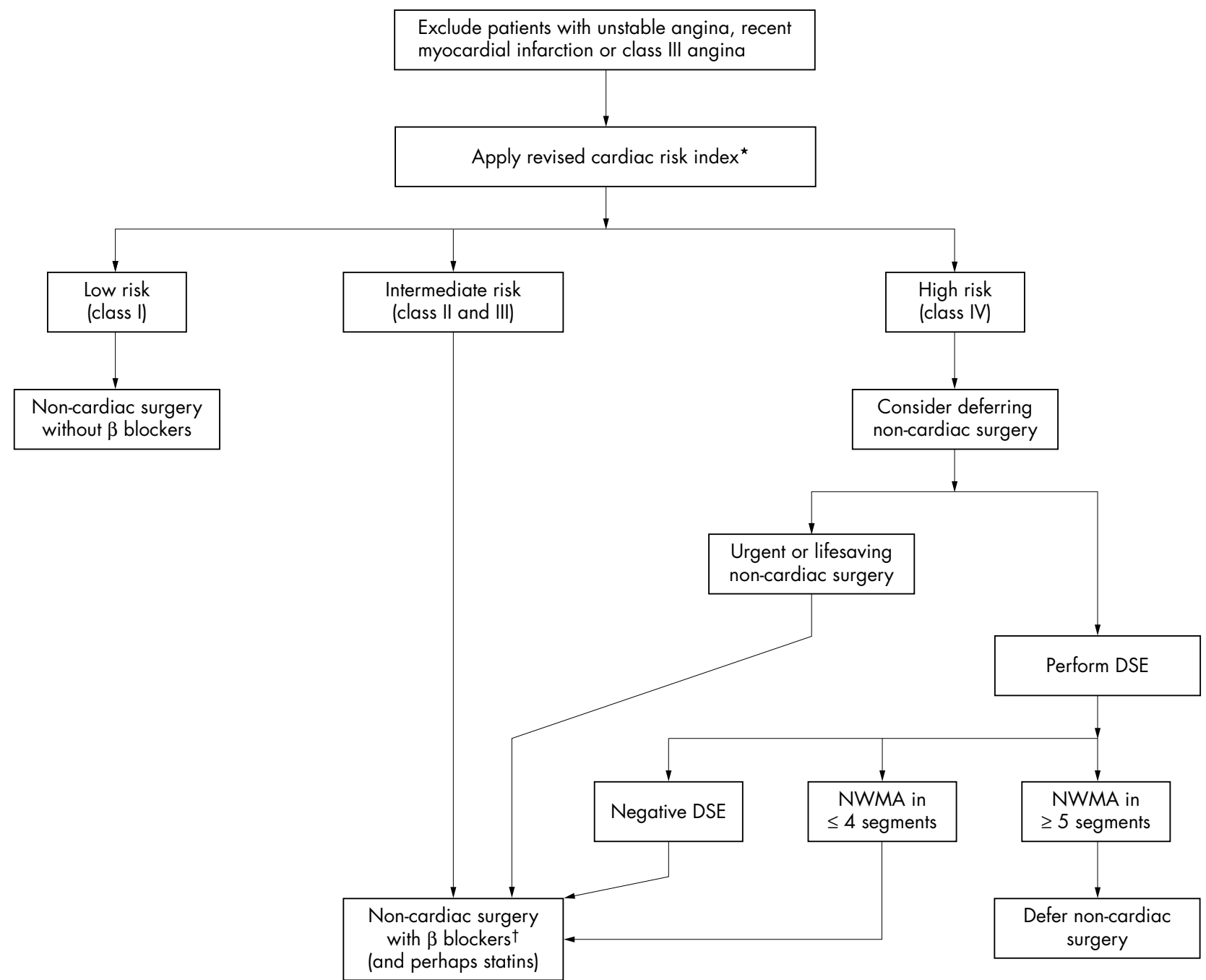

Figure 1 Proposed algorithm for the management of patients undergoing non-cardiac surgery. ${ }^{*}$ Coronary artery bypass surgery within the last five years by itself does not qualify as history of ischaemic heart disease. th the presence of contraindications to $\beta$ blocker treatment, deferring or cancellation of non-cardiac surgery should be considered, especially in patients who are class III or IV by the revised risk index. DSE, dobutamine stress echocardiography; NWMA, new wall motion abnormalities. 
stabilisation with statins or other innovative treatments ${ }^{27}$ needs to be explored. Two retrospective studies have recently shown that patients undergoing vascular surgery, who were treated with statins, had fewer perioperative cardiac events. $^{28} 29$ A small prospective, randomised study from Brazil also showed a reduction in cardiovascular events at six months after the index vascular surgery. ${ }^{30}$ Larger, randomised studies are needed to determine the statin dose and duration of treatment, and to ascertain its role in specifically preventing perioperative events as against events occurring over an extended time period.

\section{PROPOSED ALGORITHM FOR PERIOPERATIVE MANAGEMENT: KEEPING IT SIMPLE}

There is little doubt that in the presence of unstable angina, recent MI or class III angina, the non-cardiac surgical procedure should be deferred or cancelled if possible, and the evaluation and treatment of CAD should take precedence. All other patients should be evaluated by a clinical risk stratification tool like the revised cardiac risk index (fig l). Patients at low or moderate risk (class II and III of the revised risk index) should undergo non-cardiac surgery under the cover of perioperative $\beta$ blocker treatment and possibly statins. In the absence of risk factors (class I), patients can safely undergo non-cardiac surgery without the need for $\beta$ blockers. In patients categorised as high risk (class IV), if delaying non-cardiac surgery is thought to be detrimental, non-cardiac surgery with perioperative $\beta$ blocker treatment (and perhaps statins) should be advised. In the presence of contraindications to $\beta$ blocker treatment, deferring or cancellation of surgery should be strongly considered. An alternative strategy for patients who are categorised as high risk (class IV) involves performing DSE. A negative DSE predicts a low risk of cardiac events. Patients with new wall motion abnormalities (NWMA) in up to four segments can still undergo surgery if $\beta$ blockers can be given. Patients who have NWMA in five or more segments probably have a risk profile similar to those with class III or unstable angina and, clinical situation permitting, should undergo further evaluation and treatment of CAD (fig 1).

\section{CONCLUSIONS}

In the absence of evidence showing the usefulness of revascularisation before non-cardiac surgery, performing non-invasive stress testing for risk stratification appears illogical. Instead, perioperative $\beta$ blocker treatment, which is effective in preventing cardiac events over a broad spectrum of risk, and perhaps treatment with statins, should be more widely applied. The small proportion of patients who are at high risk of perioperative events can reliably be identified by clinical risk stratification tools. Further management of these patients lies in the realm of physician judgment and patient choice, until the results of prospective trials of prior coronary revascularisation or other treatments to stabilise atherosclerotic plaques rapidly become available.

\section{Authors' affiliations \\ G Karthikeyan, B Bhargava, Department of Cardiology, All India Institute of Medical Sciences, New Delhi, India \\ Competing interests: none}

GK wishes to thank Professor KR Sethuraman for introducing him to Brer Rabbit and Tar-Baby

\section{REFERENCES}

1 Goldman L, Caldera DL, Nussbaum SR, et al. Multifactorial index of cardiac risk in noncardiac surgical procedures. N Engl J Med 1977;297:845-50.
2 Lee TH, Marcantonio ER, Mangione CM, et al. Derivation and prospective validation of a simple index for prediction of cardiac risk of major noncardiac surgery. Circulation 1999; 100:1043-49.

3 Boersma E, Poldermans D, Bax JJ, et al for the DECREASE Study Group. Predictors of cardiac events after major vascular surgery: role of clinical characteristics, dobutamine echocardiography, and beta-blocker therapy. JAMA 2001;285:1865-73.

4 Eagle KA, Berger PB, Calkins $\mathrm{H}$, et al. ACC/AHA guideline update for perioperative cardiovascular evaluation for noncardiac surgery-executive summary. J Am Coll Cardiol 2002;39:542-53

5 Bodenheimer MM. Noncardiac surgery in the cardiac patient: what is the question? Ann Intern Med 1996;124:763-6.

6 Mold JW, Stein HF. The cascade effect in the clinical care of patients. N Engl J Med 1986;314:512-14.

7 Ober KP. Uncle Remus and the cascade effect in clinical medicine: Brer Rabbit kicks the Tar-Baby. Am J Med 1987;82:1009-13.

8 Sisson JC, Schoonmaker EB, Ross JC. Clinical decision analysis: the hazard of using additional data. JAMA 1976;236:1259-63.

9 Grayburn PA, Hillis DL. Cardiac events in patients undergoing noncardiac surgery: shifting the paradigm from noninvasive risk stratification to therapy. Ann Intern Med 2003;138:506-11.

10 Palda VA, Detsky AS. Perioperative assessment and management of risk from coronary artery disease. Ann Intern Med 1997;127:313-28.

11 Lee TH, Boucher CA. Clinical practice: noninvasive tests in patients with stable coronary artery disease. N Engl J Med 2001;344:1840-5.

12 Fuster V, Badimon L, Badimon JJ, et al. The pathogenesis of coronary artery disease and the acute coronary syndromes (1). N Engl J Med 1992;326:242-50.

13 Dawood MM, Gutpa DK, Southern J, et al. Pathology of fatal perioperative myocardial infarction: implications regarding pathophysiology and prevention. Int J Cardiol 1996;57:37-44.

14 Cohen MC, Aretz TH. Histological analysis of coronary artery lesions in fatal postoperative myocardial infarction. Cardiovasc Pathol 1999;8:133-9.

15 Mangano DT, Hollenberg M, Fegert G, et al. Perioperative myocardial ischemia in patients undergoing noncardiac surgery - I: Incidence and severity during the 4 day perioperative period. The study of perioperative ischemia (SPI) research group. J Am Coll Cardiol 1991;17:843-50.

16 Ellis SG, Hertzer NR, Young JR, et al. Angiographic correlates of cardiac death and myocardial infarction complicating major nonthoracic vascular surgery. Am J Cardiol 1996;77:1126-8.

17 Massie BM, Mangano DT. Assessment of perioperative risk: have we put the cart before the horse. J Am Coll Cardiol 1993;21:1353-6.

18 Mason JJ, Owens DK, Harris RA, et al. The role of coronary angiography and coronary revascularization before noncardiac vascular surgery. JAMA 1995;273:1919-25

19 Eagle KA, Rihal CS, Mickel MC, et al. Cardiac risk of noncardiac surgery: influence of coronary disease and type of surgery in 3368 operations. Circulation 1997;96:1882-7.

20 Foster ED, Davis KB, Carpenter JA, et al. Risk of noncardiac operation in patients with defined coronary disease: the coronary artery surgery study (CASS) registry experience. Ann Thorac Surg 1986;41:42-50.

21 Posner KL, Van Norman GA, Chan V. Adverse cardiac outcomes after noncardiac surgery in patients with prior percutaneous transluminal coronary angioplasty. Anesth Analg 1999;89:553-60.

22 Kaluza GL, Joseph J, Lee JR, et al. Catastrophic outcomes of noncardiac surgery soon after coronary stenting. J Am Coll Cardiol 2000;35:1288-94.

23 McFadden EP, Stabile E, Regar E, et al. Late thrombosis in drug-eluting coronary stents after discontinuation of antiplatelet therapy. Lancet 2004;364:1519-21.

24 McFalls EO, Ward HB, Moritz TE, et al. Coronary-artery revascularization before elective major vascular surgery. N Engl J Med 2004;351:2795-804

25 Mangano DT, Layug EL, Wallace A, et al. The multicenter study of perioperative ischemia research group. Effect of atenolol on mortality and cardiovascular morbidity affer noncardiac surgery. N Engl J Med 1996;335:1713-21.

26 Poldermans D, Boersma E, Bax JJ, et al. The effect of bisoprolol on perioperative mortality and myocardial infarction in high-risk patients undergoing vascular surgery. Dutch echocardiographic cardiac risk evaluation applying stress echocardiography study group. N Engl J Med 1999:341:1789-94

27 Nissen SE, Tsunoda T, Tuzcu EM, et al. Effect of recombinant ApoA-I Milano on coronary atherosclerosis in patients with acute coronary syndromes: a randomized controlled trial. JAMA 2003;290:2292-300.

28 O'Neil-Callahan K, Katsimaglis G, Tepper MR, et al. Statins decrease perioperative cardiac complications in patients undergoing noncardiac vascular surgery: the statins for risk reduction in surgery (StaRRS) study. J Am Coll Cardiol 2005;45:336-42.

29 Kertai MD, Boersma E, Westerhout CM, et al. A combination of statins and beta-blockers is independently associated with a reduction in the incidence of perioperative mortality and nonfatal myocardial infarction in patients undergoing abdominal aortic aneurysm surgery. Eur J Vasc Endovasc Surg 2004;28:343-52.

30 Durazzo AE, Machado FS, Ikeoka DT, et al. Reduction in cardiovascular events after vascular surgery with atorvastatin: a randomized trial. I Vasc Surg 2004;39:967-75. 